\title{
Rees coextensions of finite tomonoids and free pomonoids
}

\author{
Milan Petrík ${ }^{1,2} \cdot$ Thomas Vetterlein ${ }^{3}$
}

Received: 25 January 2018 / Accepted: 4 September 2018 / Published online: 28 September 2018

(c) The Author(s) 2018

\begin{abstract}
A totally ordered monoid, or tomonoid for short, is a monoid endowed with a compatible total order. We reconsider in this paper the problem of describing the one-element Rees coextensions of a finite, negative tomonoid $S$, that is, those tomonoids that are by one element larger than $S$ and whose Rees quotient by the poideal consisting of the two smallest elements is isomorphic to $S$. We show that any such coextension is a quotient of a pomonoid $\mathcal{R}(S)$, called the free one-element Rees coextension of $S$. We investigate the structure of $\mathcal{R}(S)$ and describe the relevant congruences. We moreover introduce a finite family of finite quotients of $\mathcal{R}(S)$ from which the coextensions arise in a particularly simple way.
\end{abstract}

Keywords Totally ordered monoid · Tomonoid $\cdot$ Rees congruence $\cdot$ Rees coextension · Free pomonoid · Finite-valued logic

\section{Introduction}

In fuzzy logic, the conjunction is typically interpreted by a binary operation making the chain of truth values into an integral residuated chain [6]. In case that only finitely many truth degrees are used, this means that we deal with finite, negative tomonoids. It is these latter structures that we investigate in the present paper. We recall that a tomonoid is a monoid endowed with a compatible total order $[3,4]$ and negativity means that the identity is the top element. We note that we do not assume commutativity.

Communicated by Jimmie D. Lawson.

$凶$ Thomas Vetterlein

Thomas.Vetterlein@jku.at

Milan Petrík

petrikm@tf.czu.cz; petrik@cs.cas.cz

1 Department of Mathematics, Faculty of Engineering, Czech University of Life Sciences, Prague, Czech Republic

2 Institute of Computer Science, Czech Academy of Sciences, Prague, Czech Republic

3 Department of Knowledge-Based Mathematical Systems, Johannes Kepler University, Linz, Austria 
Given a finite, negative tomonoid $T$, the set containing the smallest two elements is a poideal that induces a congruence on $T$. Following the terminology of semigroup theory, this is a Rees congruence. The quotient $S$ is by one element smaller than $T$. We may repeat the process, to obtain a chain of successively smaller tomonoids, ending up eventually with the trivial tomonoid, which consists of one element only. The question obviously arises how to proceed in the opposite direction: given a finite negative tomonoid $S$, we may wonder how to determine all those tomonoids $T$ that lead back to $S$ by an identification of its two smallest elements. We call such tomonoids $T$ the one-element Rees coextensions of $S$. We have dealt with the problem in our previous work [10], where we have proposed an algorithm to construct these coextensions in an effective way. Starting from the trivial tomonoid, the method defined in [10] can be applied to calculate successively all finite, negative tomonoids.

The topic of the present paper are the one-element Rees coextensions once more. We are interested this time, however, in algebraic rather than algorithmic aspects and to describe our procedure we have chosen a significantly different approach. Let $S$ be a non-trivial finite, negative tomonoid. We define first what we call the oneelement free Rees coextension of $S$. This is a pomonoid $\mathcal{R}(S)$ with the property that any one-element Rees coextension is among its quotients. The relevant congruences on $\mathcal{R}(S)$ are uniquely determined by the 0 class, hence to determine the one-element coextensions means to characterise those poideals that can assume this role. We provide a characterisation.

However, the pomonoid $\mathcal{R}(S)$ is infinite, in discrepancy with the fact that our intentions involve finite structures. The problem is solvable. The one-element Rees coextensions of $S$ can be roughly classified by the pairs $\left(\varepsilon_{l}, \varepsilon_{r}\right)$ of two idempotent elements of the original tomonoid. We speak, accordingly, of $\left(\varepsilon_{l}, \varepsilon_{r}\right)$-coextensions. For a given such pair $\left(\varepsilon_{l}, \varepsilon_{r}\right)$, we define a pomonoid $\mathcal{R}_{\varepsilon_{l}, \varepsilon_{r}}(S)$, which is a quotient of $\mathcal{R}(S)$ and which is finite. There is an effective way of determining $\mathcal{R}_{\varepsilon_{l}, \varepsilon_{r}}(S)$. Moreover, each one-element $\left(\varepsilon_{l}, \varepsilon_{r}\right)$-coextension is in turn a quotient of $\mathcal{R}_{\varepsilon_{l}, \varepsilon_{r}}(S)$. To determine the relevant congruences on $\mathcal{R}_{\varepsilon_{l}, \varepsilon_{r}}(S)$ is very simple; they correspond to downsets within a certain interval of $\mathcal{R}_{\varepsilon_{l}, \varepsilon_{r}}(S)$.

We note that the new approach has enabled us to define a clear context for several notions and constructions of [10] that seemed to be chosen ad hoc. For instance, we used in [10] the somewhat technical notion of a ramification, which was in turn based on an intermediate equivalence relation on a certain subset of the enlarged so-called tomonoid partition. In the present approach, the pomonoids $\mathcal{R}_{\varepsilon_{l}, \varepsilon_{r}}(S)$ have taken over the role that the ramification played in the previous context. Furthermore, the present work answers several issues that were left open or vague in our previous work. For instance, the characterisation of the totality of one-element coextensions that we briefly announced in [10, Remark 4.5] is now stated in a precise form. We moreover provide one necessary and one sufficient condition regarding the difficult question whether, for given elements $\varepsilon_{l}$ and $\varepsilon_{r}$, a one-element $\left(\varepsilon_{l}, \varepsilon_{r}\right)$-coextension exists at all.

The paper is structured as follows. In Sect. 2, we compile some basic facts about pomonoids, in particular those that concern congruences generated by inequalities, and we discuss free pomonoids. The lengthy Sect. 3 is devoted to the pomonoid $\mathcal{R}(S)$ and those congruences on $\mathcal{R}(S)$ that lead to one-element Rees coextensions of $S$. Section 4 describes the more specific procedure that results when assuming a pair $\left(\varepsilon_{l}, \varepsilon_{r}\right)$ to be 
associated with the coextension. Our concluding Sect. 5 contains a summary and an outlook to further work.

\section{Preliminaries}

Definition 2.1 A partially ordered monoid, or a pomonoid for short, is a structure $(S ; \leqslant, \cdot, 1)$ such that $(S ; \cdot, 1)$ is a monoid, $(S ; \leqslant)$ is a poset, and $\leqslant$ is compatible with the monoidal product, that is, for any $a, b, c, d \in S, a \leqslant b$ and $c \leqslant d$ imply $a \cdot c \leqslant b \cdot d$.

A pomonoid $S$ is called negative if the monoidal identity 1 is the top element. In case that the partial order is a chain, we refer to $S$ as a totally ordered monoid, or tomonoid for short.

Our work focuses on finite negative tomonoids. We remark that the notion "tomonoid" is taken from [3]; we do not assume, however, a tomonoid to be commutative. The notion "negative" is chosen in accordance with [3] as well. We note that in [4], the notion "negatively ordered" is used instead and that in the context of residuated structures, usually the notion "integral" is preferred [5].

As usual, we will denote the monoidal product often just by juxtaposition.

A map $\varphi: S \rightarrow T$ between pomonoids is a homomorphism if $\varphi$ is an order-preserving homomorphism of monoids. The homomorphism is called orderdetermining if the following holds: For any $c, d \in T$ such that $c \leqslant d$, there are $s_{0}, \ldots, s_{k} \in S$ such that

$$
\begin{aligned}
& c=\varphi\left(s_{0}\right), s_{0} \leqslant s_{1}, \varphi\left(s_{1}\right)=\varphi\left(s_{2}\right), s_{2} \leqslant s_{3}, \varphi\left(s_{3}\right)=\varphi\left(s_{4}\right), \ldots, \\
& s_{k-1} \leqslant s_{k}, \varphi\left(s_{k}\right)=d .
\end{aligned}
$$

If $\varphi: S \rightarrow T$ is a surjective, order-determining homomorphism of pomonoids, we call $T$ a homomorphic image of $S$.

Congruences for pomonoids are defined in a way such that quotients correspond to homomorphic images. Let us make the conditions explicit [1]. Let $(S ; \leqslant, \cdot, 1)$ be a pomonoid and let $\theta$ be a congruence of its monoidal reduct. In order to ensure that the monoid homomorphism $S \rightarrow S / \theta, a \mapsto a / \theta$ is order-preserving and orderdetermining, the partial order on $S / \theta$ must be the smallest preorder such that $a / \theta \leqslant$ $b / \theta$ whenever $a \leqslant b$. Let us denote by $\leqslant{ }_{\theta}$ the $\theta$-preorder on $S$ : for $a, b \in S$, we put $a \leqslant{ }_{\theta} b$ if there is a $\theta$-chain from $a$ to $b$, that is, if there are $s_{0}, \ldots, s_{k}, k \geqslant 0$, such that

$$
a=s_{0} \leqslant s_{1} \theta s_{2} \leqslant \cdots \leqslant s_{k-1} \theta s_{k}=b .
$$

Then the preorder induced by $\leqslant_{\theta}$ on $S / \theta$ is required to be a partial order, that is, antisymmetric. Accordingly, an equivalence relation $\theta$ on a pomonoid $S$ is called a congruence if

(1) $\theta$ is a congruence on the monoid $(S ; \cdot, 1)$ and

(2) for any $a, b \in S, a \leqslant \theta \quad b$ and $b \leqslant_{\theta} a$ imply $a \theta b$.

We readily check that congruences on pomonoids are in a one-to-one correspondence with homomorphic images. 
Note that, for a congruence $\theta$ on a pomonoid, each $\theta$-class is convex. In case of a total order, this property may replace condition (2). Indeed, in case that $S$ is a tomonoid, $\theta$ is a congruence on $S$ if and only if $\theta$ is a monoid congruence and each $\theta$-class is convex.

A particularly simple type of congruence is the following [2]. Recall that a (twosided) ideal of a monoid $S$ is a set $I \subseteq S$ such that $b \in I$ and $a, c \in S$ imply $a b, b c \in I$. Moreover, a subset $D$ of a poset $P$ is a downset if $b \in D$ and $a \leqslant b$ imply $a \in D$, and $D$ is an upset if $b \in D$ and $a \geqslant b$ imply $a \in D$. Given a pomonoid $S$, a poideal is a subset of $S$ that is both an ideal and a downset.

Lemma 2.2 Let $(S ; \cdot, \leqslant, 1)$ be a pomonoid and I a poideal. For $a, b \in S$, let $a \rho_{I} b$ if $a=b$ or $a, b \in I$. Then $\rho_{I}$ is a congruence.

Proof Disregarding the order, we may easily verify the well-known fact that $\rho_{I}$ is a monoid congruence [7]. As $I$ is a downset, it is immediate that $\rho_{I}$ is even a congruence of pomonoids [2].

We call $\rho_{I}$, as defined in Lemma 2.2, the Rees congruence induced by the poideal $I$ and the quotient, denoted by $S / I$, is the Rees quotient by $I$. Note that $S / I$ consists of the singletons $\{x\}, x \in S \backslash I$, and the poideal $I$. Following a common practice, we will assume in what follows that $S \backslash I$ is a subset of $S / I$ and we will refer to the class $I$ by (a variant of) a zero symbol, in accordance with the fact that this is an absorbing element.

Let us consider the case of a negative tomonoid $S$. As $a b, b c \leqslant b$ holds for any $a, b, c \in S$, a subset $I$ is a poideal if and only if $I$ is a downset. In particular, for any $q \in S$, the set $\{a \in S: a \leqslant q\}$ is a poideal and we shall write $S / q$ for the corresponding Rees quotient. Given a finite pomonoid $S$, we call the pomonoid $T$ a one-element Rees coextension of $S$, or one-element coextension for short, if $T$ possesses a unique atom $\alpha$ and $T / \alpha$ is isomorphic to $S$.

The aim of this paper is to describe the one-element coextensions of a finite, negative tomonoid. Note that this is an instance of the ideal extension problem for posemigroups, see, e.g., [8].

Before we start the actual discussion, some further preparations are necessary. We will need to have ways of generating congruences at hand.

Given an arbitrary binary relation $\rho$ on a pomonoid $S$, it is possible to construct the smallest pomonoid congruence containing $\rho$. This is, however, not our intention. Indeed, we do not want to consider congruences $\theta$ such that $a / \theta=b / \theta$ whenever $a \rho b$. We rather want to construct a congruence $\theta$ with the effect that, for all pairs of elements $a$ and $b$ such that $a \rho b$, we just have $a / \theta \leqslant b / \theta$.

The construction is due to Al Subaiei [1]. We outline a proof of the following proposition for later reference.

Proposition 2.3 Let $(S ; \cdot, \leqslant, 1)$ be a pomonoid and let $₫$ be any binary relation on $S$. Then there is a smallest congruence $\theta$ on $S$ such that $a / \theta \leqslant b / \theta$ whenever $a \leqslant b$.

The congruence $\theta$ has the following universal property. Let $f: S \rightarrow T$ be a homomorphism from $S$ to a further pomonoid $T$ such that, for any $a, b \in S, a \leqslant b$ implies $f(a) \leqslant f(b)$. Then there is a homomorphism $\tilde{f}: S / \theta \rightarrow T$ such that $\tilde{f}(a / \theta)=f(a)$. 
Proof (sketched) For $a, b \in S$, let $a \leqslant \Downarrow b$ if $a \leqslant b$ or there are $p_{1}, q_{1}, r_{1}, s_{1}, \ldots$, $p_{k}, q_{k}, r_{k}, s_{k} \in S, k \geqslant 1$, such that

$$
\begin{aligned}
& a \leqslant p_{1} r_{1} q_{1}, \quad r_{1} \Vdash s_{1}, \quad p_{1} s_{1} q_{1} \leqslant p_{2} r_{2} q_{2}, \\
& r_{2} \leqslant s_{2}, \quad p_{2} s_{2} q_{2} \leqslant p_{3} r_{3} q_{3}, \\
& \cdots, \\
& r_{k} \preccurlyeq s_{k}, \quad p_{k} s_{k} q_{k} \leqslant b .
\end{aligned}
$$

Furthermore, we put $a \theta b$ if $a \leqslant s b$ and $b \leqslant s a$, and we partially order the quotient $S / \theta$ by requiring that $a / \theta \leqslant b / \theta$ if $a \leqslant s b$. We readily check that $\theta$ is a pomonoid congruence. It is furthermore clear that $\theta$ is the smallest congruence with the property that $a / \theta \leqslant b / \theta$ for all $a, b \in S$ such that $a \leqslant b$. Also the remaining part is shown by standard arguments.

Given a relation $\sharp$ on a pomonoid $S$, we denote the congruence specified in Proposition 2.3 by $\Theta(\sharp)$ and we say that $\Theta(\sharp)$ is generated by $\sharp$.

We next turn to free constructions, for which we may once again refer to [1].

Proposition 2.4 Let $(G ; \leqslant)$ be a poset. Then there is a pomonoid $\mathcal{F}(G)$ and an order-preserving map $\iota: G \rightarrow \mathcal{F}(G)$ with the following universal property: for any pomonoid $T$ and order-preserving map $f: G \rightarrow T$, there is a unique pomonoid homomorphism $\bar{f}: \mathcal{F}(G) \rightarrow T$ such that $f=\bar{f} \circ \iota$.

Proof (sketched) We take $\mathcal{F}(G)$ as the monoid of words $a_{1} \ldots a_{n}$, where $n \geqslant 0$ and $a_{1}, \ldots, a_{n} \in G$. The product is defined by concatenation and the identity is given by the empty word. We partially order $\mathcal{F}(G)$ be setting

$$
a_{1} \ldots a_{m} \leqslant b_{1} \ldots b_{n} \text { if } n=m \text { and } a_{1} \leqslant b_{1}, \ldots, a_{n} \leqslant b_{n} .
$$

Finally, we let $\iota: G \rightarrow \mathcal{F}(G)$ map each $a \in G$ to itself, considered as a word of length 1 .

The pomonoid $\mathcal{F}(G)$, that is, the monoid of words over $G$ partially ordered by (2), will be called the free pomonoid on the poset $G$. We will denote its identity, the empty word, by $\varepsilon$. Note that the insertion map $\iota: G \rightarrow \mathcal{F}(G)$, which maps $a \in G$ to the corresponding word of length 1 , is an embedding of posets: we have $a \leqslant b$ in $G$ if and only if $\iota(a) \leqslant \iota(b)$ in $\mathcal{F}(G)$. In the sequel we can thus safely view $G$ as a subposet of $\mathcal{F}(G)$. Accordingly, we will say that any order-preserving map from $G$ to some pomonoid can be extended to $\mathcal{F}(G)$.

Combining Propositions 2.3 and 2.4, we see that we may specify pomonoids as quotients of free pomonoids in a common way: we indicate a poset $G$ together with a set of inequalities among the elements of $\mathcal{F}(G)$. Identifying the inequalities with a binary relation $\sharp$ on $\mathcal{F}(G)$, the pomonoid defined in this way is the quotient of the free pomonoid on $G$ induced by the congruence generated by $\sharp$, that is, $\mathcal{F}(G) / \Theta(\sharp)$. In practice, we will indicate the poset together with a set of equalities and inequalities; for, an equality $a=b$ can be identified with the two inequalities $a \leqslant b$ and $b \leqslant a$.

To avoid a cumbersome presentation, we will in this context abuse notation as follows. Unless otherwise noticed, we will make no difference in notation between 
the elements of $\mathcal{F}(G)$, and in particular the elements of $G$, on the one hand and their $\Theta(\unlhd)$-classes on the other hand. For instance, let $a \in G$; then we will usually refer to the element $a / \Theta(\sharp)$ of $\mathcal{F}(G) / \Theta(\sharp)$ by the symbol $a$ as well. It will be clear from the context what we mean.

\section{The free one-element Rees coextension}

In this section, $(S ; \cdot, \leqslant, 1)$ is a finite negative tomonoid. We are interested in the oneelement (Rees) coextensions of $S$. To this end, we will define a pomonoid of which any of the desired coextensions is a quotient.

If $S=\{1\}$, we call $S$ the trivial tomonoid. In this case, $S$ possesses exactly one one-element Rees-coextension. Indeed, the two element chain $\{0,1\}$ can be made into a tomonoid in only one way: by defining $1 \cdot 1=1$ and $0 \cdot 1=1 \cdot 0=0 \cdot 0=0$. We will assume from now on that $S$ is non-trivial, that is, $S$ possesses at least two elements.

Denoting by $\dot{0}$ the smallest element of $S$, we define $S^{\star}=S \backslash\{\dot{0}\}$ and $\bar{S}=S^{\star} \dot{\cup}\{0, \alpha\}$, where 0 and $\alpha$ are new elements. We extend the total order on $S^{\star}$ to $\bar{S}$, letting $0 \leqslant \alpha \leqslant a$ for any $a \in S^{\star}$.

We view $S^{\star}$ as a partial monoid; cf., e.g., [11]. The multiplication on $S^{\star}$ is defined partially, the product of two elements $a, b \in S^{\star}$ being defined only if $a b$, calculated in $S$, is an element of $S^{\star}$. Then $1 \in S^{\star}$, the products $1 a$ and $a 1$ exist for all $a \in S^{\star}$, and $1 a=a 1=a$. Furthermore, for $a, b, c \in S^{\star}$, the products $a b$ and $(a b) c$ exist if and only if $b c$ and $a(b c)$ exist, in which case $(a b) c=a(b c)$.

Our aim is to determine all possible ways of endowing $\bar{S}$ with a monoidal product such that $\bar{S}$ becomes a one-element coextension of $S$. Let us clarify what this means. Let $(\bar{S} ; \bullet, \leqslant, 1)$ be a finite, negative tomonoid and assume that $S$ is the Rees quotient of $\bar{S}$ by the ideal $\{0, \alpha\}$. Note that $S$ consists of the elements of $S^{\star}$, which we identify with the singleton congruence classes, and of the element $\dot{0}$, which corresponds to the congruence class $\{0, \alpha\}$. The multiplication in $S$ arises from the multiplication in $\bar{S}$ as follows: for $a, b \in S$, we have

$$
a \cdot b= \begin{cases}a \cdot b & \text { if } a, b \neq \dot{0} \text { and, in } \bar{S}, a \cdot b \notin\{0, \alpha\} \\ \dot{0} & \text { otherwise }\end{cases}
$$

In the present context, we consider $(S ; \cdot, \leqslant, 1)$ as fixed and we intend to determine $(\bar{S} ; \bullet, \leqslant, 1)$. From (3) we observe that the multiplication on $S$ determines to a good extent the one on $\bar{S}$ : for any two elements $a, b \in \bar{S} \backslash\{0, \alpha\}$ whose product is non-zero in $S$, this product is in $\bar{S}$ the same as in $S$. We may say that the partial monoid $S^{\star}$ is a substructure of $\bar{S}$ : whenever, for $a, b \in S^{\star}, a b$ is defined and equals $c$ in $S^{\star}$, we have that $a b=c$ holds in $\bar{S}$ as well. Consequently, our aim is the extension of the partial tomonoid $S^{\star}$ to a (total) tomonoid based on the chain $\bar{S}$.

For a pair $(a, b) \in \bar{S}^{2}$ such that $a, b \in S^{\star}$ and $a b$ is defined in $S^{\star}$, the product of $a$ and $b$ is in $\bar{S}$ thus determined from the outset. We denote the set of all remaining pairs of elements of $\bar{S}$ as follows:

$$
\begin{aligned}
& \mathcal{N}=\left\{(a, b) \in \bar{S}^{2}: a, b>\alpha \text { and } a b=\dot{0} \text { in } S\right\} \\
& \cup\{0, \alpha\} \times \bar{S} \cup \bar{S} \times\{0, \alpha\} .
\end{aligned}
$$


For a pair $(a, b) \in \mathcal{N}$, it is in general not clear what the product of $a$ and $b$ in $\bar{S}$ is, but it is clear that $a b$ equals either 0 or $\alpha$. Indeed, if $a, b>\alpha$, then $a b=c>\alpha$ in $\bar{S}$ would imply that we have $a b=c$ in $S$ as well and thus $a b \neq \dot{0}$ in $S$. Moreover, if $a \leqslant \alpha$ or $b \leqslant \alpha$, it follows $a b \leqslant \alpha$ by the negativity of $\bar{S}$, that is, $a b=0$ or $a b=\alpha$. Determining the coextension $\bar{S}$ means accordingly that we have to suitably divide $\mathcal{N}$ into a set of pairs mapped to 0 and a set of pairs mapped to $\alpha$.

We add that even the products of certain pairs in $\mathcal{N}$ are clear from the outset. Indeed, we have $0 a=a 0=0$ for any $a \in \bar{S}$ and $1 \alpha=\alpha 1=\alpha$. But the following examples show that in all remaining cases the product may really be either 0 or $\alpha$.

Example 3.1 For $a, b \in \bar{S}$, let us define

$$
a \cdot b= \begin{cases}a b & \text { if } a, b \in S^{\star} \text { and } a b \text { exists in } S^{\star} \\ \alpha & \text { if } a=\alpha \text { and } b=1, \text { or } a=1 \text { and } b=\alpha \\ 0 & \text { otherwise. }\end{cases}
$$

We readily verify that $(\bar{S} ; \bullet, \leqslant, 1)$ is a one-element coextension of $(S ; \cdot, \leqslant, 1)$.

Example 3.2 A further one-element coextension is $(\bar{S} ; \bullet, \leqslant, 1)$, where, for $a, b \in \bar{S}$,

$$
a \cdot b= \begin{cases}a b & \text { if } a, b \in S^{\star} \text { and } a b \text { exists in } S^{\star}, \\ 0 & \text { if } a=0 \text { or } b=0, \\ \alpha & \text { otherwise. }\end{cases}
$$

These examples are, in a sense, the extreme cases. Indeed, in case of Example 3.1, all products that are not determined from the outset are defined to be 0 , whereas in case of Example 3.2 all these are $\alpha$.

We now provide the key definition on which the present paper is based.

Definition 3.3 Let $\mathcal{R}(S)$ be the free pomonoid over the chain $\bar{S}$, subject to the following conditions:

(a) $a b=c$ for any $a, b, c \in S^{\star}$ fulfilling this equation in $S$,

(b) $\varepsilon=1$.

(c) $a b \leqslant \alpha$ for any $(a, b) \in \mathcal{N}$,

(d) $0 a=0$ for any $a \in \bar{S}$,

We call $\mathcal{R}(S)$ the free one-element Rees coextension, or simply the free one-element coextension of $S$.

Proposition 3.4 Let $T$ be a one-element coextension of $S$. Then there is a congruence $\theta$ on $\mathcal{R}(S)$ such that each $\theta$-class contains exactly one $a \in \bar{S}$ and $\mathcal{R}(S) / \theta$ is isomorphic to $T$.

Proof Note that $\bar{S}$ and $T$ are chains of equal size; let $f: \bar{S} \rightarrow T$ be the order isomorphism. By Proposition 2.4, $f$ extends to a pomonoid homomorphism $\bar{f}: \mathcal{F}(\bar{S}) \rightarrow T$.

Let us identify the equalities and inequalities (a)-(d) in Definition 3.3 with the binary relation $\sharp$ on $\mathcal{F}(\bar{S})$; for instance, by (c) we require $a b \Downarrow \alpha$ for $(a, b) \in \mathcal{N}$. 
Then $\mathcal{R}(S)=\mathcal{F}(\bar{S}) / \Theta(\Downarrow)$. It is obvious that, for any $a, b \in \mathcal{F}(\bar{S}), a \unlhd b$ implies $\bar{f}(a) \leqslant \bar{f}(b)$. Hence, by Proposition 2.3, there is a homomorphism $\tilde{\bar{f}}: \mathcal{R}(S) \rightarrow T$ such that $\tilde{\bar{f}}(a / \Theta(\varangle))=\bar{f}(a)$ for any $a \in \mathcal{F}(\bar{S})$. In particular, for $a \in \bar{S}$, we have $\tilde{\bar{f}}(a / \Theta(\unlhd))=f(a)$ and it follows that $\tilde{\bar{f}}$ is order-determining.

Thus $T$ is a homomorphic image of $\mathcal{R}(S)$ and the assertions follow.

To shorten the subsequent statements, let us call a congruence on $\mathcal{R}(S)$ that, in the way indicated in Proposition 3.4, leads to a one-element coextension of $S$, a coextension congruence. Our aim is therefore to characterise these particular congruences on $\mathcal{R}(S)$.

We begin by describing $\mathcal{R}(S)$ itself. From now on we will make use of the simplified notation announced earlier: an element of $\bar{S}$ or a word in $\mathcal{F}(\bar{S})$ will also denote its congruence class in $\mathcal{R}(S)$.

Proposition 3.5 Let $\beta$ be the bottom element of $S^{\star}$, that is, the atom of $S$.

(i) $\mathcal{R}(S)$ is the union of the intervals $[0, \alpha]$ and $[\beta, 1]$, and we have $0<\alpha<\beta$. Moreover, $[0, \alpha]$ is a poideal and $[\beta, 1]$ is a chain, consisting of the pairwise distinct elements $a \in S^{\star}$.

(ii) 1 is the top element and the identity of $\mathcal{R}(S)$; in particular, $\mathcal{R}(S)$ is a negative pomonoid.

(iii) Let $a, b \in[\beta, 1]$. If $a b=c$ holds in $S^{\star}$, then we have $a b=c$ in $\mathcal{R}(S)$ as well. If $a b$ is in $S^{\star}$ undefined, then we have $a b \leqslant \alpha$ in $\mathcal{R}(S)$.

Proof We first show that the elements of $\bar{S}$ are in $\mathcal{R}(S)$ pairwise distinct. Indeed, for any one-element coextension $T$ there is by Proposition 3.4 a congruence $\theta$ on $\mathcal{R}(S)$ such that $a \in \bar{S}$ are all in distinct classes and $T$ is isomorphic to the quotient. Moreover, by Examples 3.1 and 3.2, a one-element coextension always exists. We conclude that distinct elements of $\bar{S}$ are indeed distinct in $\mathcal{R}(S)$.

By the definition of $\mathcal{R}(S)$, the elements of $\bar{S}$ moreover form a chain in $\mathcal{R}(S)$. In particular, we have $0<\alpha<\beta \leqslant 1$, thus $[0, \alpha]$ and $[\beta, 1]$ are disjoint intervals of $\mathcal{R}(S)$.

We have $\varepsilon=1$ by the defining equality (b) of Definition 3.3, thus 1 is the identity of $\mathcal{R}(S)$. We furthermore have $a \leqslant 1$ for any $a \in \bar{S}$, and $1 \cdot 1=1$. For $a_{1}, \ldots, a_{k} \in \bar{S}$, $k \geqslant 1$, it follows $a_{1} \ldots a_{k} \leqslant 1 \cdots 1=1$. Hence 1 is the top element of $\mathcal{R}(S)$.

Furthermore, equality (d) of Definition 3.3 and the fact that $0 \leqslant a$ for any $\bar{S}$ imply that 0 is the bottom element of $\mathcal{R}(S)$. Hence $[0, \alpha]$ is a downset and since $\mathcal{R}(S)$ is negative, $[0, \alpha]$ is actually a poideal.

Let now $a, b \in S^{\star}$. Then $a, b \in[\beta, 1]$. Assume first that in $S$ we have $a b=c \neq \dot{0}$. This means $a b=c$ holds in $S^{\star}$ and by equality (a) the same equality then holds in $\mathcal{R}(S)$. Assume second that in $S$ we have $a b=\dot{0}$. This means that $a b$ is in $S^{\star}$ undefined and by inequality (c) we have $a b \leqslant \alpha$.

As $\mathcal{R}(S)$ is (as a monoid) generated by $\bar{S}$ and $[0, \alpha]$ is a poideal, we conclude that $[\beta, 1]$ consists of the elements of $S^{\star}$ only and $\mathcal{R}(S)=[0, \alpha] \cup[\beta, 1]$.

We thus see that the free one-element coextension $\mathcal{R}(S)$ consists of $S^{\star}$ and, strictly below this set, the poideal $[0, \alpha]$. We note that the latter set is infinite. Proposition 3.5 furthermore implies that $\mathcal{R}(S) /[0, \alpha]$ is (isomorphic to) $S$. 
Assume that $\theta$ is a coextension congruence. Then each $\theta$-class contains exactly one $a \in \bar{S}$. Hence, by Proposition 3.5, the quotient consists of the singletons $a / \theta=\{a\}$, $a \in S^{\star}$, as well as $0 / \theta$ and $\alpha / \theta$, which partition $[0, \alpha]$. In particular, a coextension congruence is uniquely determined by the class $0 / \theta$.

Proposition 3.6 Let $Z \subseteq[0, \alpha]$ be a downset of $\mathcal{R}(S)$ that is non-empty but does not contain $\alpha$. Assume that the following condition holds:

For any $a, b \in[0, \alpha] \backslash Z$ and any $c \in \bar{S}, a c \in Z$ if and only if $b c \in Z$, and $c a \in Z$ if and only if $c b \in Z$.

For $a, b \in \mathcal{R}(S)$, let

$$
a \theta_{Z} b \text { if and only if } a=b \text { or } a, b \in Z \text { or } a, b \in[0, \alpha] \backslash Z \text {. }
$$

Then $\theta_{Z}$ is a congruence on $\mathcal{R}(S)$ such that $Z=0 / \theta_{Z}$, and $\mathcal{R}(S) / \theta_{Z}$ is a one-element coextension of $S$.

Up to isomorphism, every one-element coextension of $S$ arises in this way from a unique downset $Z$ of $\mathcal{R}(S)$.

Proof Let $Z$ be a downset of $[0, \alpha]$ and let $\theta_{Z}$ be given as indicated. The $\theta_{Z}$-classes are then the singletons $\{a\}$ for each $a \in S^{\star}$, as well as $Z$ and $[0, \alpha] \backslash Z$. To show that $\theta_{Z}$ is a congruence, let $a, b, c \in \mathcal{R}(S)$. Assume that $a \theta_{Z} b$; we have to show that $a c \theta_{Z} b c$. Obviously, we may restrict to the case $c \in \bar{S}$. If $a \in S^{\star}$, then $a=b$ and the assertion is clear. If $a \in Z$, then also $b \in Z$, and because $Z$ is an ideal, we have $a c, b c \in Z$ and hence $a c \theta_{Z} b c$. Let $a \in[0, \alpha] \backslash Z$. Then also $b \in[0, \alpha] \backslash Z$. Because $[0, \alpha]$ is a poideal, we have that $a c, b c \in[0, \alpha]$. By assumption, $a c$ and $b c$ are either both in $Z$ or both in $[0, \alpha] \backslash Z$, thus $a c \theta_{Z} b c$. A similar argument shows that also $c a \theta_{Z} c b$ holds, and we conclude that $\theta_{Z}$ is a monoid congruence.

To see that $\theta_{Z}$ is in fact a pomonoid congruence, assume $c_{0} \leqslant c_{1} \theta_{Z} c_{2} \leqslant \ldots \theta_{Z} c_{k}=$ $c_{0}$. As $Z$ and $[0, \alpha]$ are downsets, it follows that $c_{0}, \ldots, c_{k}$ all lie in the same $\theta_{Z}$-class.

Consider now the Rees quotient of $\mathcal{R}(S) / \theta_{Z}$ by the two-element poideal $\left[0 / \theta_{Z}\right.$, $\left.\alpha / \theta_{Z}\right]$. The resulting tomonoid is obviously isomorphic to $\mathcal{R}(S) /[0, \alpha]$ and hence to $S$. This means that $\mathcal{R}(S) / \theta_{Z}$ is a one-element coextension of $S$.

We now turn to the last assertion. Let $T$ be a one-element coextension of $S$. By Proposition 3.4, there is a congruence $\theta$ on $\mathcal{R}(S)$ such that $\mathcal{R}(S) / \theta$ is isomorphic with $T$. Identifying $T$ with $\bar{S}$, the elements of $T$ are the classes $a / \theta, a \in \bar{S}$, and we have $a / \theta=\{a\}$ for $a \in S^{\star}$ and $0 / \theta \cup \alpha / \theta=[0, \alpha]$.

Let $Z=0 / \theta$. As 0 is the bottom element of $\mathcal{R}(S)$ and the $\theta$-classes are convex, $Z$ is a downset of $\mathcal{R}(S)$ such that $\varnothing \subset Z \subset[0, \alpha]$. Furthermore, we have $[0, \alpha] \backslash Z=\alpha / \theta$. It follows that $\theta=\theta_{Z}$ according to (6). Moreover, for any $a, b \in[0, \alpha] \backslash Z$, we have $a \theta b \theta \alpha$ and hence $a c \theta b c \theta \alpha c$. Since $\alpha c \in[0, \alpha]$ it follows that $a c$ and $b c$ are both in $Z$ or both in $[0, \alpha] \backslash Z$. This completes the proof that all one-element coextensions of $S$ arise in the way asserted from a downset $Z$ of $\mathcal{R}(S)$.

It remains to show that the downset giving rise to a one-element coextension is unique. But this is clear from the fact that distinct downsets $Z \subset[0, \alpha]$ induce distinct congruences $\theta$, that is, distinct products on $\mathcal{R}(S) / \theta$. 


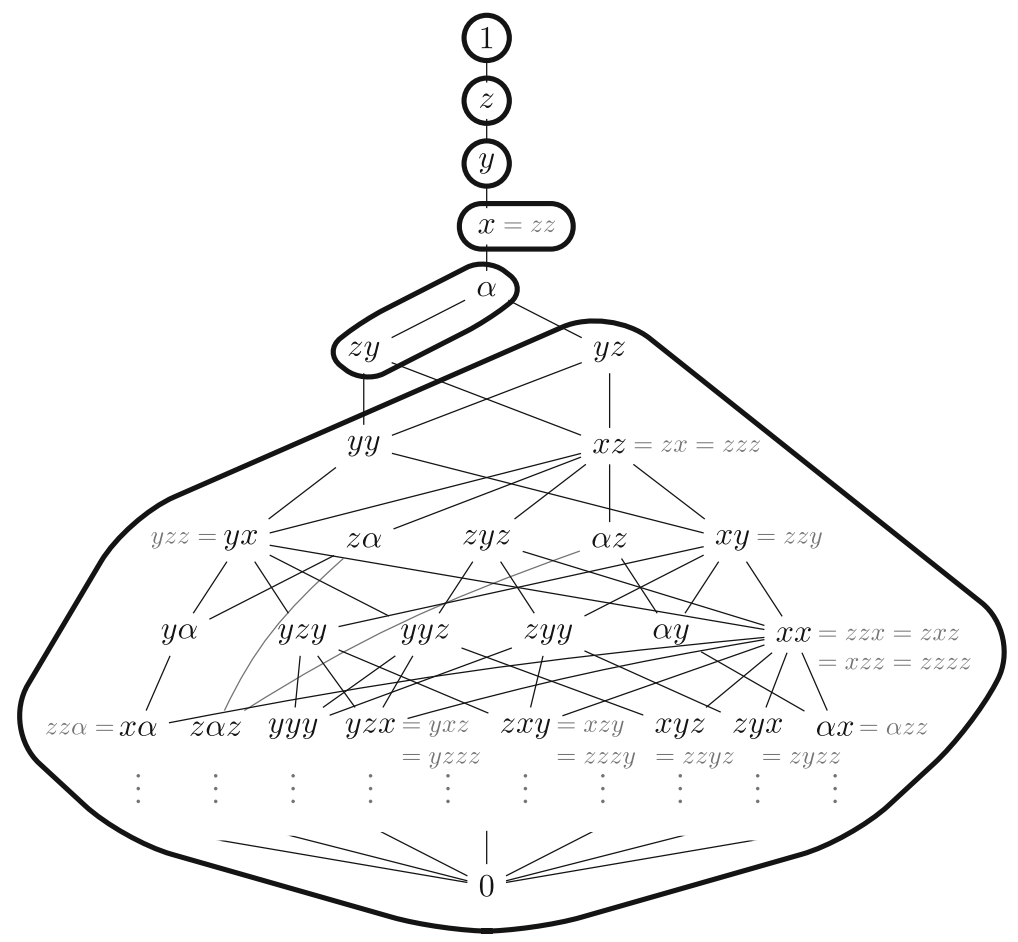

Fig. 1 The free one-element coextension $\mathcal{R}(S)$ for the tomonoid $S$ from Example 3.7 and a coextension congruence

Example 3.7 We consider an example illustrating Proposition 3.6. Here as well as in case of subsequent examples of tomonoids, we denote the elements of the base set by lower case Latin letters from the end of the alphabet, except for the bottom and top elements, which are denoted by $\dot{0}$ and 1, respectively. Let $S$ be the five-element chain, that is, let

$$
S=\{\dot{0}, x, y, z, 1\}
$$

understood to be totally ordered as indicated. We make $S$ into a tomonoid by defining, for $a, b \in S$,

$$
a \cdot b= \begin{cases}b & \text { if } a=1 \\ a & \text { if } b=1 \\ x & \text { if } a=b=z \\ \dot{0} & \text { otherwise }\end{cases}
$$

The free one-element coextension $\mathcal{R}(S)$ is infinite; Fig. 1 shows a part of its poset reduct. Furthermore, an example of a coextension congruence is indicated; the congruence classes are encircled. 
According to Proposition 3.6, it would now be natural to aim at a characterisation of the downset $Z=0 / \theta$, where $\theta$ is a coextension congruence. Although this is feasible, we proceed more conveniently as follows.

Lemma 3.8 Let $\theta$ be a coextension congruence on $\mathcal{R}(S)$ and define

$$
\mathcal{Z}_{\theta}=\left\{(a, b) \in \bar{S}^{2}: a b \theta 0\right\} .
$$

Then $\mathcal{Z}_{\theta} \subseteq \mathcal{N}$. Moreover, $\theta$ is the congruence generated by the equations

$$
a b=0 \text { for any }(a, b) \in \mathcal{Z}_{\theta}, \quad a b=\alpha \text { for any }(a, b) \in \mathcal{N} \backslash \mathcal{Z}_{\theta} .
$$

Proof If $(a, b) \in \bar{S}^{2} \backslash \mathcal{N}$, then $a b \in S^{\star}$ and hence $(a, b) \notin \mathcal{Z}_{\theta}$. That is, $\mathcal{Z}_{\theta} \subseteq \mathcal{N}$.

The only $\theta$-classes that are not singletons are $0 / \theta$ and $\alpha / \theta$. Let $a=a_{1} \ldots a_{k} \in$ $\mathcal{R}(S)$, where $a_{1}, \ldots, a_{k} \in \bar{S}, k \geqslant 0$. If $k=0$, then $a=1$ and hence neither $a \theta 0$ nor $a \theta \alpha$ holds. If $k=1$, then $a \theta 0$ iff $a=0$, and $a \theta \alpha$ iff $a=\alpha$. Let $k=2$. Then $a \theta 0$ iff $\left(a_{1}, a_{2}\right) \in \mathcal{Z}_{\theta}$ and $a \theta \alpha$ iff $\left(a_{1}, a_{2}\right) \in \mathcal{N} \backslash \mathcal{Z}_{\theta}$. Finally, assume that $k \geqslant 3$. Let $a_{1} \ldots a_{k-1} \theta z$, where $z \in \bar{S}$. Then again, $a \theta 0$ iff $\left(z, a_{k}\right) \in \mathcal{Z}_{\theta}$, and $a \theta \alpha$ iff $\left(z, a_{k}\right) \in \mathcal{N} \backslash \mathcal{Z}_{\theta}$. Hence the assertion follows by an inductive argument.

We conclude that each coextension congruence $\theta$ on $\mathcal{R}(S)$ is determined by the set $\mathcal{Z}_{\theta} \subseteq \mathcal{N}$. The following lemma makes explicit how the class $0 / \theta$ is determined by $\mathcal{Z}_{\theta}$.

Lemma 3.9 Let $\theta$ be a coextension congruence on $\mathcal{R}(S)$. Let $a=a_{1} \ldots a_{k} \in[0, \alpha]$, where $a_{1}, \ldots, a_{k} \in \bar{S}, k \geqslant 1$. Let $i \in\{1, \ldots, k\}$ be smallest such that the product $a_{1} \ldots a_{i}$ is not in $S^{\star}$. We have a $\theta 0$ if and only if either $a_{i}=0$, or $i<k$ and $\left(\alpha, a_{j}\right) \in \mathcal{Z}_{\theta}$ for some $i<j \leqslant k$, or $i \geqslant 2$ and $\left(a_{1} \ldots a_{i-1}, a_{i}\right) \in \mathcal{Z}_{\theta}$.

Proof Let $i$ be as indicated; then $a_{1} \ldots a_{i} \theta 0$ or $a_{1} \ldots a_{i} \theta \alpha$. It is easily checked that $a \theta 0$ holds under one of the indicated conditions.

Conversely, assume $a \theta 0$. If $a_{1} \ldots a_{i} \theta 0$, then either $i=1$ and $a_{i}=a_{1}=0$, or $i \geqslant 2$ and $\left(a_{1} \ldots a_{i-1}, a_{i}\right) \in \mathcal{Z}_{\theta}$. If $a_{1} \ldots a_{i} \theta \alpha$, then there is a smallest $j \in\{i+1, \ldots k\}$ such that $a_{1} \ldots a_{j-1} \theta \alpha$, thus $a_{1} \ldots a_{j} \theta 0$ and consequently $\left(\alpha, a_{j}\right) \in \mathcal{Z}_{\theta}$.

Our aim is the characterisation of the set $\mathcal{Z}_{\theta} \subseteq \mathcal{N}$. We need some preparations.

Definition 3.10 Let $\theta$ be a coextension congruence on $\mathcal{R}(S)$. Then we call the smallest element $\varepsilon_{l} \in S^{\star} \cup\{\alpha\}$ such that $\varepsilon_{l} \alpha \theta \alpha$ the left border for $\theta$. Similarly, we call the smallest element $\varepsilon_{r} \in S^{\star} \cup\{\alpha\}$ such that $\alpha \varepsilon_{r} \theta \alpha$ the right border for $\theta$.

We will denote the left and right border for a coextension congruence $\theta$ in the sequel always by $\varepsilon_{l}$ and $\varepsilon_{r}$, respectively. The name is justified by the fact that $\varepsilon_{l}$ and $\varepsilon_{r}$ provide a limitation for the class $0 / \theta$ and hence for $\mathcal{Z}_{\theta}$, as stated in the following remark.

Remark 3.11 Let $\theta$ be a coextension congruence on $\mathcal{R}(S)$. For any $a \in \bar{S}$, we have $0=0 \alpha \leqslant a \alpha \leqslant 1 \alpha=\alpha$ and thus either $a \alpha \theta 0$ or $a \alpha \theta \alpha$. Hence $\varepsilon_{l}$ is the smallest 
element in $\bar{S}$ such that $\left(\varepsilon_{l}, \alpha\right) \notin \mathcal{Z}_{\theta}$, that is, for $a \in \bar{S}$ we have $(a, \alpha) \in \mathcal{Z}_{\theta}$ iff $a<\varepsilon_{l}$. Similarly we may characterise $\varepsilon_{r}$.

Note furthermore that, for any $a, b \in \bar{S}$ such that $(a, b) \in \mathcal{Z}_{\theta}$, we have $a=0$, or $b=0$, or $a<\varepsilon_{l}$ and $b<\varepsilon_{r}$.

Lemma 3.12 Let $\theta$ be a coextension congruence with the borders $\varepsilon_{l}, \varepsilon_{r}$.

(i) $\varepsilon_{l}=\alpha$ if and only if $\varepsilon_{r}=\alpha$. In this case, $0 / \theta=\{0\}$ and the quotient is isomorphic to $(\bar{S} ; \bullet, \leqslant, 1)$ from Example 3.2. In particular, $\alpha / \theta$ is idempotent.

(ii) Let $\varepsilon_{l}, \varepsilon_{r} \in S^{\star}$. Then both $\varepsilon_{l}$ and $\varepsilon_{r}$ are idempotent elements of $S$.

Proof (i) Assume that $\varepsilon_{l}=\alpha$. This means $\alpha^{2} \theta \alpha$ and hence also $\varepsilon_{r}=\alpha$. Similarly, we see that $\varepsilon_{r}=\alpha$ implies $\varepsilon_{l}=\alpha$.

Moreover, for any $a, b \in \bar{S} \backslash\{0\}$, we have in this case $a b / \theta=a / \theta \cdot b / \theta \geqslant$ $(\alpha / \theta)^{2}=\alpha / \theta$. It follows $0 / \theta=\{0\}$ and multiplication in $\mathcal{R}(S) / \theta$ is given according to (5).

(ii) By definition, $\varepsilon_{l} \alpha \theta \alpha$ and hence $\varepsilon_{l}^{2} \alpha \theta \varepsilon_{l} \alpha \theta \alpha$. We claim that $\varepsilon_{l}^{2} \in S^{\star}$. Indeed, otherwise $\varepsilon_{l}^{2} \leqslant \alpha<\varepsilon_{l}$ would imply $\varepsilon_{l}^{2} \alpha \leqslant \alpha^{2} \theta 0$, a contradiction. From the minimality property of $\varepsilon_{l}$ we conclude $\varepsilon_{l} \leqslant \varepsilon_{l}^{2}$. As we have $\varepsilon_{l}^{2} \leqslant \varepsilon_{l}$ by the negativity of $\mathcal{R}(S)$, we have that $\varepsilon_{l}^{2}=\varepsilon_{l}$ holds in $\mathcal{R}(S)$ and hence also in $S$.

The assertion concerning $\varepsilon_{r}$ is seen analogously.

We conclude that the border elements $\varepsilon_{l}, \varepsilon_{r}$ either both equal $\alpha$ or are idempotent elements of the partial monoid $S^{\star}$.

Note that since $S^{\star}=S \backslash\{\dot{0}\}$ and $\dot{0}$ is an idempotent element of $S$, we can identify the pair $\varepsilon_{l}, \varepsilon_{r}$ with a pair of two idempotent elements of the original tomonoid $S$; in this case, $\dot{0} \in S$ corresponds to $\alpha \in \bar{S}$.

We are now ready to compile the characteristic properties of the set $\mathcal{Z}_{\theta}$.

Lemma 3.13 Let $\theta$ be a coextension congruence on $\mathcal{R}(S)$ with the borders $\varepsilon_{l}, \varepsilon_{r}$. Then $\mathcal{Z}=\mathcal{Z}_{\theta}$ is a subset of $\mathcal{N}$ such that, for any $a, b, c, d \in \bar{S}$, the following holds:

(Z1) If $(b, d) \in \mathcal{Z}$ and $a \leqslant b, c \leqslant d$, then $(a, c) \in \mathcal{Z}$.

(Z2) $(a, 0),(0, b) \in \mathcal{Z}$ and $(\alpha, 1),(1, \alpha) \notin \mathcal{Z}$.

(Z3) Let $a b, b c \in S^{\star}$. Then $(a, b c) \in \mathcal{Z}$ if and only if $(a b, c) \in \mathcal{Z}$.

(Z4) If $(a, b),(b, c) \in \mathcal{N}, a \geqslant \varepsilon_{l}$, and $c<\varepsilon_{r}$, then $(b, c) \in \mathcal{Z}$.

If $(a, b),(b, c) \in \mathcal{N}, a<\varepsilon_{l}$, and $c \geqslant \varepsilon_{r}$, then $(a, b) \in \mathcal{Z}$.

(Z5) Let $b c \in S^{\star}$ or $b=1$. If $(a, b) \in \mathcal{N}, c<\varepsilon_{r}$, then $(a, b c) \in \mathcal{Z}$.

Let $a b \in S^{\star}$ or $b=1$. If $(b, c) \in \mathcal{N}, a<\varepsilon_{l}$, then $(a b, c) \in \mathcal{Z}$.

(Z6) Let $b c \in S^{\star}$ or $b=1$. If $(a, b) \in \mathcal{N}, c \geqslant \varepsilon_{r}$, and $(a, b c) \in \mathcal{Z}$, then $(a, b) \in \mathcal{Z}$. Let $a b \in S^{\star}$ or $b=1$. If $(b, c) \in \mathcal{N}, a \geqslant \varepsilon_{l}$, and $(a b, c) \in \mathcal{Z}$, then $(b, c) \in \mathcal{Z}$.

Proof (Z1), (Z2), and (Z3) are immediate from the definition of $\mathcal{Z}_{\theta}$.

In case of (Z4)-(Z6), we show the first halves only; the second ones are seen similarly.

Ad (Z4): Assume that $(a, b),(b, c) \in \mathcal{N}, a \geqslant \varepsilon_{l}$, and $c<\varepsilon_{r}$. Then $a b \leqslant \alpha$ and hence $a b c \theta 0$ by the definition of $\varepsilon_{r}$. Moreover, $b c \leqslant \alpha$ and hence $b c \theta 0$ or $b c \theta \alpha$. In the latter case, it would follow $a b c \theta \alpha$ by the definition of $\varepsilon_{l}$, hence $b c \theta 0$. 
Ad (Z5): Assume that $(a, b) \in \mathcal{N}$ and $c<\varepsilon_{r}$. Then $a b c \theta 0$ and hence $(a, b c) \in \mathcal{Z}$.

$\operatorname{Ad}\left(\right.$ Z6): Assume that $(a, b) \in \mathcal{N}$ and $c \geqslant \varepsilon_{r}$. Then $(a, b) \in \mathcal{N} \backslash \mathcal{Z}$ implies $a b c \theta \alpha$ and hence $(a, b c) \in \mathcal{N} \backslash \mathcal{Z}$.

We now turn to the converse direction: given a pair $\varepsilon_{l}, \varepsilon_{r}$ of border elements, we describe the possible subsets $\mathcal{Z}_{\theta}$ of $\mathcal{N}$ that determine a coextension congruence $\theta$.

Lemma 3.14 Let $\varepsilon_{l}, \varepsilon_{r} \in S^{\star} \cup\{\alpha\}$ and let $\mathcal{Z} \subseteq \mathcal{N}$ be such that, for any $a, b, c, d \in$ $\bar{S}$, the conditions (Z1)-(Z6) hold. Then there is a uniquely determined coextension congruence $\theta$ with the borders $\varepsilon_{l}, \varepsilon_{r}$ such that $\mathcal{Z}=\mathcal{Z}_{\theta}$.

Proof Before beginning the proof, let us show two additional properties of $\mathcal{Z}$.

(Z7) For any $(a, b) \in \mathcal{Z}$ such that $a, b \neq 0$, we have $a<\varepsilon_{l}$ and $b<\varepsilon_{r}$.

Indeed, we may apply the second half of (Z6) to $b=1$ and $c=\alpha$, to conclude that $(a, \alpha) \notin \mathcal{Z}$ if $a \geqslant \varepsilon_{l}$. In view of (Z1), this shows one half of (Z7). The other one is seen similarly.

(Z8) $(a, \alpha) \in \mathcal{Z}$ for any $a<\varepsilon_{l}$, and $(a, \alpha) \in \mathcal{N} \backslash \mathcal{Z}$ for any $a \geqslant \varepsilon_{l}$. Similarly, $(\alpha, b) \in \mathcal{Z}$ for any $b<\varepsilon_{r}$, and $(\alpha, b) \in \mathcal{N} \backslash \mathcal{Z}$ for any $b \geqslant \varepsilon_{r}$.

Indeed, applying the second half of (Z5) to $b=1$ and $c=\alpha$, we see that $(a, \alpha) \in \mathcal{Z}$ if $a<\varepsilon_{l}$. Furthermore, if $a \geqslant \varepsilon_{l}$, then $(a, \alpha) \notin \mathcal{Z}$ by (Z7). This shows one half of (Z8); the other one is seen similarly.

For $a, b \in \bar{S}$, let us define

$$
a \cdot b= \begin{cases}a b & \text { if } a, b, a b \in S^{\star} \\ 0 & \text { if }(a, b) \in \mathcal{Z}, \\ \alpha & \text { if }(a, b) \in \mathcal{N} \backslash \mathcal{Z}\end{cases}
$$

We shall show that the operation $\bullet$ makes $\bar{S}$ into a tomonoid. Indeed, 1 is an identity for $\bullet$, and it is clear that $\bullet$ is in both arguments order-preserving. It remains to prove the associativity.

Let $a, b, c \in \bar{S}$; we have to show that $(a \bullet b) \bullet c=a \bullet(b \bullet c)$. We distinguish several cases.

Case 1. Assume that $a b c \in S^{\star}$. Then $a b, b c \in S^{\star}$ and hence $(a \bullet b) \bullet c=a b c=$ $a \cdot(b \cdot c)$.

Case 2. Assume that $a b, b c \in S^{\star}$ but $a b c \notin S^{\star}$. Then $(a b, c),(a, b c) \in \mathcal{N}$ and, by (Z3), $(a \cdot b) \cdot c=a b \cdot c=a \cdot b c=a \cdot(b \cdot c)$.

Case 3. Assume that $(a, b) \in \mathcal{Z}$. Then $(a \cdot b) \cdot c=0 \cdot c=0$ by (Z2). Note, furthermore, that $b \bullet c \leqslant b$. Hence $(a, b \bullet c) \in \mathcal{Z}$ by (Z1) and we conclude $a \bullet(b \bullet c)=0$.

Case 4. Assume that $(b, c) \in \mathcal{Z}$. We proceed analogously to Case 3 .

Case 5. Assume that $(a, b),(b, c) \in \mathcal{N} \backslash \mathcal{Z}$. If $a<\varepsilon_{l}$ and $c \geqslant \varepsilon_{r}$, or $a \geqslant \varepsilon_{l}$ and $c<\varepsilon_{r}$, we get a contradiction by (Z4). Hence either $a<\varepsilon_{l}$ and $c<\varepsilon_{r}$, or $a \geqslant \varepsilon_{l}$ and $c \geqslant \varepsilon_{r}$. From (Z8), we derive $(a \cdot b) \cdot c=\alpha \cdot c=a \cdot \alpha=a \cdot(b \cdot c)$.

Case 6. Assume that $(a, b) \in \mathcal{N} \backslash \mathcal{Z}$ and $b c \in S^{\star}$. If $c<\varepsilon_{r}$, we have $(a \bullet b) \bullet c=$ $\alpha \cdot c=0$ and, by (Z5), $a \cdot(b \cdot c)=a \cdot b c=0$ as well. If $c \geqslant \varepsilon_{r}$, we have $(a \cdot b) \cdot c=\alpha \cdot c=\alpha$ and, by (Z6), $a \cdot(b \cdot c)=a \cdot b c=\alpha$ as well. 6.

Case 7. Assume that $(b, c) \in \mathcal{N} \backslash \mathcal{Z}$ and $a b \in S^{\star}$. We proceed analogously to Case 
We conclude that $(\bar{S} ; \leqslant, \bullet, 1)$ is a tomonoid. Furthermore, $\bar{S} / \alpha$ is isomorphic to $S$, that is, $\bar{S}$ is a one-element coextension of $S$. Let $\theta$ be the coextension congruence on $\mathcal{R}(S)$ such that $\bar{S}$ is isomorphic to $\mathcal{R}(S) / \theta$. Then, for any $(a, b) \in \bar{S}^{2}$, we have

$$
(a, b) \in \mathcal{Z}_{\theta} \text { iff } a b \theta 0 \text { iff } a / \theta \cdot b / \theta=0 / \theta \text { iff } a \cdot b=0 \text { in } \bar{S} \text { iff }(a, b) \in \mathcal{Z} .
$$

Hence $\mathcal{Z}=\mathcal{Z}_{\theta}$. By (Z8), the border elements of $\theta$ are $\varepsilon_{l}, \varepsilon_{r}$.

Finally, let $\theta^{\prime}$ be another coextension congruence on $\mathcal{R}(S)$ such that $\mathcal{Z}=\mathcal{Z}_{\theta^{\prime}}$. But then $\mathcal{Z}_{\theta}=\mathcal{Z}_{\theta^{\prime}}$ and hence, by Lemma 3.8, $\theta$ and $\theta^{\prime}$ coincide. The uniqueness claim follows.

By a one-element $\left(\varepsilon_{l}, \varepsilon_{r}\right)$-coextension of $S$, we mean the quotient of $\mathcal{R}(S)$ induced by a coextension congruence with borders $\varepsilon_{l}, \varepsilon_{r} \in S^{\star} \cup\{\alpha\}$. We may summarise our results as follows.

Theorem 3.15 Let $\varepsilon_{l}, \varepsilon_{r} \in S^{\star} \cup\{\alpha\}$ and let $\mathcal{Z} \subseteq \mathcal{N}$ be such that, for any $a, b, c, d \in S^{\star}$, the conditions (Z1)-(Z6) hold. For $a, b \in \bar{S}$, let

$$
a \cdot b= \begin{cases}a b & \text { if } a, b, a b \in S^{\star} \\ 0 & \text { if }(a, b) \in \mathcal{Z}, \\ \alpha & \text { if }(a, b) \in \mathcal{N} \backslash \mathcal{Z} .\end{cases}
$$

Then $(\bar{S} ; \leqslant, \bullet, 1)$ is a one-element $\left(\varepsilon_{l}, \varepsilon_{r}\right)$-coextension of $S$.

Up to isomorphism, any one-element $\left(\varepsilon_{l}, \varepsilon_{r}\right)$-coextension arises in this way from a unique set $\mathcal{Z} \subseteq \bar{S}^{2}$.

Proof The first part holds by Lemma 3.14; the second part holds by Lemma 3.13.

In short, for a pair $\varepsilon_{l}, \varepsilon_{r} \in S^{\star} \cup\{\alpha\}$, there is a one-to-one correspondence between the one-element $\left(\varepsilon_{l}, \varepsilon_{r}\right)$-coextensions of $S$ and the sets $\mathcal{Z} \subseteq \mathcal{N}$ subject to the conditions (Z1)-(Z6).

We can formulate Theorem 3.15 as follows in an algorithmic fashion. At this point, the present work becomes comparable with the approach chosen in [10].

In what follows, we endow $\mathcal{N}$ with the componentwise partial order $\sharp$, that is, for $(a, b),(c, d) \in \mathcal{N}$, we write $(a, b) \leqslant(c, d)$ if $a \leqslant b$ and $c \leqslant d$.

Theorem 3.16 Let $\varepsilon_{l}, \varepsilon_{r} \in S^{\star} \cup\{\alpha\}$ such that that either $\varepsilon_{l}=\varepsilon_{r}=\alpha$ or $\varepsilon_{l}, \varepsilon_{r}$ are idempotent elements of $S^{\star}$. Let $\sim$ be the smallest equivalence relation on $\mathcal{N}$ such that following holds:

(A1) For any $a, b \in \bar{S}$, we have $(a, 0) \sim(0, b) \sim(1,0)$. Moreover, for any $a \in \bar{S}$ such that $a<\varepsilon_{l}$, we have $(a, \alpha) \sim(1,0)$. Similarly, for any $a \in \bar{S}$ such that $a<\varepsilon_{r}$, we have $(\alpha, a) \sim(1,0)$.

(A2) We have $(1, \alpha) \sim(\alpha, 1)$ and for any $(a, b) \in \mathcal{N}$ such that $a, b \neq 0$ and $a \geqslant \varepsilon_{l}$ or $b \geqslant \varepsilon_{r}$, we have $(a, b) \sim(1, \alpha)$.

(A3) Let $a, b, c, a b, b c \in S^{\star}$ and $(a, b c),(a b, c) \in \mathcal{N}$. Then $(a, b c) \sim(a b, c)$.

(A4) Let $(a, b),(b, c) \in \mathcal{N}$ such that $a \geqslant \varepsilon_{l}$ and $c<\varepsilon_{r}$. Then $(b, c) \sim(1,0)$.

Similarly, let $(a, b),(b, c) \in \mathcal{N}$ such that $a<\varepsilon_{l}$ and $c \geqslant \varepsilon_{r}$. Then $(a, b) \sim(1,0)$. 


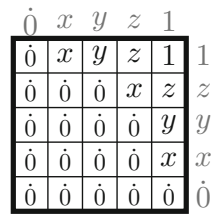

0
\begin{tabular}{|c|c|c|c|c|c|c|c|}
\hline 0 & $\alpha$ & $x$ & $y$ & $z$ & 1 & 1 \\
\hline 0 & 0 & $A$ & $C$ & $x$ & $z$ & $z$ \\
\hline 0 & 0 & 0 & $B$ & $D$ & $y$ & $y$ \\
\hline 0 & 0 & 0 & 0 & $A$ & $x$ & $x$ \\
\hline 0 & 0 & 0 & 0 & 0 & $\alpha$ & $\alpha$ \\
\hline 0 & 0 & 0 & 0 & 0 & 0 & 0
\end{tabular}

Fig. 2 The coextension of $S$, where $S$ is from Example 3.7. Left: The multiplication table of $S$. Right: The classes of the equivalence relation $\sim$ on $\bar{S}$

(A5) Let $a, b, c, b c \in S^{\star}$, $(a, b) \in \mathcal{N}$, and $c<\varepsilon_{r}$. Then $(a, b c) \sim(1,0)$. Similarly, let $a, b, c, a b \in S^{\star},(b, c) \in \mathcal{N}$, and $a<\varepsilon_{l}$. Then $(a b, c) \sim(1,0)$.

(A6) Let $a, b, c, b c \in S^{\star},(a, b) \in \mathcal{N}$, and $c \geqslant \varepsilon_{r}$. Then $(a, b) \sim(a, b c)$. Similarly, let $a, b, c, a b \in S^{\star},(b, c) \in \mathcal{N}$, and $a \geqslant \varepsilon_{l}$. Then $(b, c) \sim(a b, c)$.

If $(1,0) \sim(1, \alpha)$, a one-element $\left(\varepsilon_{l}, \varepsilon_{r}\right)$-coextension of $S$ does not exist. Otherwise, let $\mathcal{Z} \subseteq \mathcal{N}$ be a union of $\sim$-classes such that $(1,0) \in \mathcal{Z},(1, \alpha) \notin \mathcal{Z}$, and $\mathcal{Z}$ is a downset. Then $(\bar{S} ; \bullet, \leqslant, 1)$, where $\bullet$ is defined by $(7)$, is a one-element $\left(\varepsilon_{l}, \varepsilon_{r}\right)$ coextension of $S$.

Every one-element coextension of $S$ arises in this way.

Proof Assume $(1,0) \nsim(1, \alpha)$ and let $\mathcal{Z} \subseteq \mathcal{N}$ arise in the indicated fashion. Then $\mathcal{Z}$ fulfils the properties (Z1)-(Z6) of Lemma 3.13; this is not difficult to check and we omit the details. Consequently, $\mathcal{Z}$ gives rise to a one-element coextension as indicated in Theorem 3.15.

Conversely, let $\mathcal{Z}=\mathcal{Z}_{\theta}$, where $\theta$ is a coextension congruence. Then $\mathcal{Z}$ is a subset of $\mathcal{N}$ that fulfils, by Lemma 3.13 and the proof of Lemma 3.14, the properties (Z1)-(Z8). By (Z2), $(1,0) \in \mathcal{Z}$ but $(1, \alpha) \notin \mathcal{Z}$. It is now easily checked that, for any $a, b, c, d \in \bar{S}$ such that $(a, b) \sim(c, d)$, either $(a, b),(c, d) \in \mathcal{Z}$ or $(a, b),(c, d) \notin \mathcal{Z}$. Hence $\mathcal{Z}$ is a union of $\sim$-classes. Since $\mathcal{Z}$ contains $(1,0)$ but not $(1, \alpha)$, we have $(1,0) \nsim(1, \alpha)$. Finally, we have by (Z1) that $(c, d) \in \mathcal{Z}, a \leqslant c, b \leqslant d$ imply $(a, b) \in \mathcal{Z}$, that is, $\mathcal{Z}$ is a downset. We conclude that any one-element coextension arises in the indicated way.

Example 3.17 Let us consider again the tomonoid $S$ indicated in Example 3.7. Its multiplication table is given in Fig. 2(left). It is understood that the rows correspond to the first argument and the columns to the second one.

We determine the one-element coextensions of $S$ for the case $\varepsilon_{l}=\varepsilon_{r}=1$. The extended base set is $\bar{S}=\{0, \alpha, x, y, z, 1\}$. In accordance with Theorem 3.16, we have to calculate the equivalence relation $\sim$ on $\mathcal{N} \subseteq \bar{S}^{2}$.

The $\sim$-classes are indicated in Fig. 2(right). Here as well as in the subsequent figures, $\mathcal{N}$ is delimited against the remaining elements of $\bar{S}^{2}$ by a bold line. Moreover, we denote the $\sim$-class containing $(1,0)$ by 0 , the $\sim$-class containing $(1, \alpha)$ by $\alpha$, and the remaining ones by Latin capital letters. It is indicated which element of $\mathcal{N}$ belongs to which $\sim$-class. We see that $\mathcal{N}$ contains, apart from the classes 0 and $\alpha$, the four further classes $A, B, C$, and $D$.

By Theorem 3.16, a one-element coextension of $S$ corresponds to a union $\mathcal{Z}$ of $\sim$-classes, including the class 0 but excluding $\alpha$ and such that $(c, d) \in \mathcal{Z}, a \leqslant c$, and 
$b \leqslant d$ imply $(a, b) \in \mathcal{Z}$. For instance, let $\mathcal{Z}$ be the union of the classes $0, A, B$, and $D$. Then we arrive at the coextension $(\bar{S} ; \leqslant, \bullet, 1)$ that we have indicated in Fig. 1 . The multiplication on $\bar{S}$ is in this case defined as follows:

$$
a \cdot b= \begin{cases}b & \text { if } a=1, \\ a & \text { if } b=1, \\ x & \text { if } a=b=z, \\ \alpha & \text { if } a=z \text { and } b=y \\ 0 & \text { otherwise }\end{cases}
$$

where $a, b \in \bar{S}$.

\section{One-element coextensions for given borders}

Again, we fix in this section a non-trivial finite negative tomonoid $(S ; \cdot, \leqslant, 1)$. We have specified in the previous section a method to determine the one-element coextensions of $S$. Recall that $\mathcal{N}$ consists of those pairs of elements of the extended base set $\bar{S}$ whose product is not defined in the partial monoid $S^{\star}$. Then the subsets of $\mathcal{N}$ that are subject to certain properties are in a one-to-one correspondence with the desired coextensions.

The one-element coextensions are roughly classified by the border elements $\varepsilon_{l}, \varepsilon_{r} \in$ $S^{\star} \cup\{\alpha\}$ for the corresponding congruence on $\mathcal{R}(S)$. Here we reconsider the procedure of the previous section, but this time we assume a choice of border elements from the outset. Accordingly, let also $\varepsilon_{l}, \varepsilon_{r} \in S^{\star} \cup\{\alpha\}$ be fixed in this section, such that either $\varepsilon_{l}=\varepsilon_{r}=\alpha$ or $\varepsilon_{l}, \varepsilon_{r}$ are idempotent elements of $S^{\star}$.

Definition 4.1 Let $\mathcal{R}_{\varepsilon_{l}, \varepsilon_{r}}(S)$ be the free pomonoid over the chain $\bar{S}$, subject to the conditions (a)-(d) of Definition 3.3 as well as the following ones, for any $a, b, c \in \bar{S}$ :

(e) $a b c=b c$ for any $(b, c) \in \mathcal{N}$ and $a \geqslant \varepsilon_{l}$.

$a b c=a b$ for any $(a, b) \in \mathcal{N}$ and $c \geqslant \varepsilon_{r}$.

(f) $a b c=0$ for any $(b, c) \in \mathcal{N}$ and $a<\varepsilon_{l}$.

$a b c=0$ for any $(a, b) \in \mathcal{N}$ and $c<\varepsilon_{r}$.

We call $\mathcal{R}_{\varepsilon_{l}, \varepsilon_{r}}(S)$ the free one-element Rees coextension of $S$ w.r.t. $\left(\varepsilon_{l}, \varepsilon_{r}\right)$, or simply the free one-element $\left(\varepsilon_{l}, \varepsilon_{r}\right)$-coextension of $S$.

Proposition 4.2 Let $T$ be a one-element $\left(\varepsilon_{l}, \varepsilon_{r}\right)$-coextension of $S$. Then there is a congruence $\theta$ on $\mathcal{R}(S)$ such that each $\theta$-class contains exactly one $a \in \bar{S}$ and $\mathcal{R}(S) / \theta$ is isomorphic to $T$.

Proof This is seen similarly as Proposition 3.4.

We note that a one-element $\left(\varepsilon_{l}, \varepsilon_{r}\right)$-coextension of $S$ may not exist. We will discuss this point at the end of this section.

Lemma 4.3 In $\mathcal{R}_{\varepsilon_{l}, \varepsilon_{r}}(S)$, the following holds, for any $a, b, c \in \bar{S}$ : 
(g) $a \alpha=0$ for any $a<\varepsilon_{l}$.

$\alpha b=0$ for any $b<\varepsilon_{r}$.

(h) $a b=\alpha$ for any $(a, b) \in \mathcal{N}$ such that $a, b \neq 0$, and $a \geqslant \varepsilon_{l}$ or $b \geqslant \varepsilon_{r}$.

Proof Ad (g): Let $a<\varepsilon_{l}$. Since $(\alpha, 1) \in \mathcal{N}$, we have by (f) $a \cdot \alpha=a \cdot \alpha \cdot 1=0$. This is the first part of $(\mathrm{g})$; the second one is seen similarly.

Ad (h): Let $a \geqslant \varepsilon_{l}$ and $b \neq 0$. Since $(\alpha, 1) \in \mathcal{N}$, we have by (e) that $a \cdot \alpha=$ $a \cdot \alpha \cdot 1=\alpha \cdot 1=\alpha$. Moreover, $b \in \bar{S} \backslash\{0\}$, that is, $b \geqslant \alpha$ and hence $a \cdot b \geqslant \alpha$. By (c), $a \cdot b \leqslant \alpha$ if $(a, b) \in \mathcal{N}$. The first part of (h) is shown; similarly we see the second part.

We continue with a description of $\mathcal{R}_{\varepsilon_{l}, \varepsilon_{r}}(S)$.

Proposition 4.4 Let $\beta$ be the bottom element of $S^{\star}$, that is, the atom of $S$.

(i) $\mathcal{R}_{\varepsilon_{l}, \varepsilon_{r}}(S)$ is the union of the intervals $[0, \alpha]$ and $[\beta, 1]$, and we have $\alpha<\beta$. $[0, \alpha]$ is a poideal and equals $\{a b:(a, b) \in \mathcal{N}\}$. Moreover, $[\beta, 1]$ is a chain, consisting of the pairwise distinct elements $a \in S^{\star}$.

In particular, $\mathcal{R}_{\varepsilon_{l}, \varepsilon_{r}}(S)$ is finite.

(ii) 1 is the top element and the identity of $\mathcal{R}_{\varepsilon_{l}, \varepsilon_{r}}(S)$; in particular, $\mathcal{R}_{\varepsilon_{l}, \varepsilon_{r}}(S)$ is a negative pomonoid.

(iii) Let $a, b \in[\beta, 1]$. If $a b=c$ holds in $S^{\star}$, then we have $a b=c$ in $\mathcal{R}_{\varepsilon_{l}, \varepsilon_{r}}(S)$ as well. If $a b$ is in $S^{\star}$ undefined, then we have $a b \leqslant \alpha$ in $\mathcal{R}_{\varepsilon_{l}, \varepsilon_{r}}(S)$.

Proof $\mathcal{R}_{\varepsilon_{l}, \varepsilon_{r}}(S)$ is a quotient of $\mathcal{R}(S)$, being subjected to the additional equalities (e) and (f). The latter involve elements of $[0, \alpha]$ only. Hence most statements follow from Proposition 3.5.

What remains to show is $[0, \alpha]=\{a b:(a, b) \in \mathcal{N}\}$. We will actually show that, in $\mathcal{R}_{\varepsilon_{l}, \varepsilon_{r}}(S)$, each word of length 3 equals a word of length 2 . Let $a, b, c \in \bar{S}$. If one of $a, b$, or $c$ equals 0 , we have $a b c=0$ by equality (d). If one of $a, b$, or $c$ equals $\alpha, a b c$ equals 0 or $\alpha$ according to equalities (g) and (h). Let $a, b, c \in S^{\star}$. If $a b=d \in S^{\star}$, we have $a b c=d c$ by equality (a). If $(a, b) \in \mathcal{N}$, we have by equality (f) that $a b c=0$ if $c<\varepsilon_{r}$, and by equality (e) $a b c=a b$ otherwise. The assertion follows.

We see that $\mathcal{R}_{\varepsilon_{l}, \varepsilon_{r}}(S)$ consists of the elements $a \in S^{\star}$ as well as those of the form $a b$, where $(a, b) \in \mathcal{N}$. Moreover, the multiplication among elements in $S^{\star}$ is like in $S$ if the result is in $S^{\star}$ again, and the multiplication of elements of the form $a b$, $(a, b) \in \mathcal{N}$, with any $c \in \bar{S}$, as well as the multiplication of $\alpha$ with any $c \in \bar{S}$, is given by equalities (e)-(h).

In order to determine $\mathcal{R}_{\varepsilon_{1}, \varepsilon_{r}}(S)$ it is consequently necessary to know which among the elements $a b$, where $(a, b) \in \mathcal{N}$, coincide. It turns out that the corresponding partition of $\mathcal{N}$ is essentially the one occurring in Theorem 3.16.

Recall that $\mathcal{N}$ is endowed with the componentwise order $\sharp$. Given an equivalence relation $\sim$ on $\mathcal{N}$, let $\Downarrow_{\sim}$ be the smallest preorder on $\mathcal{N}$ such that $(a, b) \sharp_{\sim}(c, d)$ if $(a, b) \preccurlyeq(c, d)$ or $(a, b) \sim(c, d)$ or $(c, d)=(1, \alpha)$. Furthermore, let $(a, b) \approx_{\sim}(c, d)$ if $(a, b) \sharp_{\sim}(c, d)$ and $(c, d) \sharp_{\sim}(a, b)$.

Theorem 4.5 Let $\sim$ be the equivalence relation on $\mathcal{N}$ specified in Theorem 3.16. Then, for any $(a, b),(c, d) \in \mathcal{N}$, we have $(a, b) \approx \sim(c, d)$ if and only if $a b=c d$ in $\mathcal{R}_{\varepsilon_{l}, \varepsilon_{r}}(S)$. 
Proof We first consider the "only if" part. Our first aim is to show that, for any $(a, b),(c, d) \in \mathcal{N}$ such that $(a, b) \sim(c, d)$, we have $a b=c d$.

We have to check the conditions (A1)-(A6) of Theorem 3.16. We omit those cases that are seen similarly to those shown.

(A1) For $a, b \in \bar{S}$, we have $a \cdot 0=0 \cdot b=0=1 \cdot 0$. If $a<\varepsilon_{l}$, we have $a \cdot \alpha=0=1 \cdot 0$ by $(\mathrm{g})$.

(A2) We have $1 \cdot \alpha=\alpha \cdot 1$. For $(a, b) \in \mathcal{N}$ such that $a, b \neq 0$ and $a \geqslant \varepsilon_{l}$ or $b \geqslant \varepsilon_{r}$, we have $a b=\alpha$ by (h).

(A3) Let $(a, b c),(a b, c) \in \mathcal{N}$. Obviously the products of the two entries are in both cases the same.

(A4) Let $(a, b),(b, c) \in \mathcal{N}$ such that $a \geqslant \varepsilon_{l}$ and $c<\varepsilon_{r}$. Then $a b \leqslant \alpha$ and, by (g), $\alpha c=0$, hence $a b c=0$. Moreover, by (e), $a b c=b c$. We conclude $b c=0$.

(A5) Let $(a, b) \in \mathcal{N}$ and $c<\varepsilon_{r}$. Then, by (f), $a b c=0$.

(A6) Let $(a, b) \in \mathcal{N}$ and $c \geqslant \varepsilon_{r}$. Then, by (e), $a b c=a b$.

It follows that, for $(a, b),(c, d) \in \mathcal{N},(a, b) \sim(c, d)$ implies $a b=c d$. Moreover, $(a, b) \leqslant(c, d)$ implies $a b \leqslant c d$, and we have $a b \leqslant \alpha$ for any $(a, b) \in \mathcal{N}$. The proof of the "only if" part is complete.

To see the "if" part, assume that $(a, b),(c, d) \in \mathcal{N}$ are such that $(a, b) \Downarrow_{\sim}(c, d)$ does not hold. Let then $\mathcal{Z}=\left\{(s, t) \in \mathcal{N}:(s, t) \sharp_{\sim}(c, d)\right\}$. Then $\mathcal{Z}$ is a union of $\sim$-classes and a downset, and we have $(c, d) \in \mathcal{Z}$ but $(a, b) \notin \mathcal{Z}$. Furthermore, $(1,0) \in \mathcal{Z}$ because $(1,0) \sim(0,0) \preccurlyeq(c, d)$. But $(1, \alpha) \notin \mathcal{Z}$. Indeed, otherwise we would have $(1, \alpha) \sharp_{\sim}(c, d)$, and since $(a, b) \sharp_{\sim}(1, \alpha)$ it would follow $(a, b) \sharp_{\sim}(c, d)$, contrary to our assumption. Note in particular that $(1, \alpha) \nsim(1,0)$.

By Theorem 3.16, there is a one-element $\left(\varepsilon_{l}, \varepsilon_{r}\right)$-coextension of $S$ such that the products $a \cdot b$ and $c \cdot d$ are different. It follows $a b \neq c d$ in $\mathcal{R}_{\varepsilon_{l}, \varepsilon_{r}}(S)$.

By Theorem 4.5, we are now in the position to determine $\mathcal{R}_{\varepsilon_{l}, \varepsilon_{r}}(S)$ in an effective way. We start with the equivalence relation $\sim$ on $\mathcal{N}$, as described by Theorem 3.16. Then we identify those $\sim$-classes forming $₫$-cycles and if necessary we enlarge the class of $(1, \alpha)$ to an upset of $\mathcal{N}$. The resulting $\approx_{\sim}$-classes can be considered as the elements of the poideal $[0, \alpha]$ of $\mathcal{R}_{\varepsilon_{l}, \varepsilon_{r}}(S)$; for each pair $(a, b) \in \mathcal{N}, a b$ is the element of $[0, \alpha]$ associated with the $\approx_{\sim}$-class of $(a, b)$. Moreover, the multiplication in $\mathcal{R}_{\varepsilon_{l}, \varepsilon_{r}}(S)$ is given by the product of $S$ as regards $a, b \in S^{\star}$ such that $a b \in S^{\star} ; 0$ is an absorbing element; and all remaining cases are covered by equalities (e)-(h).

To illustrate this procedure, we insert several examples.

Example 4.6 We consider once more the tomonoid $S$ from Examples 3.7 and 3.17. We want to determine $\mathcal{R}_{1,1}(S)$. To this end, we have to calculate the equivalence relation $\approx \sim$ on $\mathcal{N}$. Figure 2 (right) shows $\sim$ and we see that there are no cycles w.r.t. $\sharp$. Hence $\approx \sim$ coincides with $\sim$. Here and in the two following examples, we denote the elements of $[0, \alpha] \subseteq \mathcal{R}_{1,1}(S)$ by the associated subsets of $\mathcal{N}$. Then $\mathcal{R}_{1,1}(S)=$ $\{0, A, B, C, D, \alpha, x, y, z, 1\}$. The multiplication and the order is specified in Fig. 3.

By Theorem 4.10 , the one-element $(1,1)$-coextensions of $S$ correspond to the downsets $J$ of the six-element interval $[0, \alpha]$ such that $0 \in J$ but $\alpha \notin J$. There are seven in total. 
Fig. 3 The free one-element $(1,1)$-coextension $\mathcal{R}_{1,1}(S)$ discussed in Example 4.6. Here, $S$ is again the tomonoid from Example 3.7
For $a, b \in \mathcal{R}_{1,1}(S)$, we have

$$
a \cdot b= \begin{cases}b & \text { if } a=1, \\ a & \text { if } b=1 \\ x & \text { if } a=b=z \\ A & \text { if } a=z \text { and } b=x \\ & \text { or } a=x \text { and } b=z \\ B & \text { if } a=b=y \\ C & \text { if } a=z \text { and } b=y \\ D & \text { if } a=y \text { and } b=z \\ 0 & \text { otherwise }\end{cases}
$$

$\dot{0}$\begin{tabular}{ll|l|l|l|l|l|l|}
\hline$\dot{0}$ & $v$ & $w$ & $x$ & $y$ & $z$ & 1 & 1 \\
\hline$\dot{0}$ & $\dot{0}$ & $v$ & $v$ & $v$ & $x$ & $z$ & $z$ \\
\hline$\dot{0}$ & $\dot{0}$ & $\dot{0}$ & $\dot{0}$ & $\dot{0}$ & $v$ & $y$ & $y$ \\
\hline$\dot{0}$ & $\dot{0}$ & $\dot{0}$ & $\dot{0}$ & $\dot{0}$ & $v$ & $x$ & $x$ \\
\hline$\dot{0}$ & $\dot{0}$ & $\dot{0}$ & $\dot{0}$ & $\dot{0}$ & $v$ & $w$ & $w$ \\
\hline$\dot{0}$ & $\dot{0}$ & $\dot{0}$ & $\dot{0}$ & $\dot{0}$ & $\dot{0}$ & $v$ & $v$ \\
\hline$\dot{0}$ & $\dot{0}$ & $\dot{0}$ & $\dot{0}$ & $\dot{0}$ & $\dot{0}$ & $\dot{0}$ & $\dot{0}$ \\
\hline
\end{tabular}

0 \begin{tabular}{l|l|l|l|l|l|l|l|l|}
\hline 0 & $\alpha$ & $w$ & $x$ & $y$ & $z$ & 1 \\
\hline 0 & $\alpha$ & $v$ & $w$ & $x$ & $y$ & $z$ & 1 & 1 \\
\hline 0 & 0 & $A$ & $v$ & $v$ & $v$ & $x$ & $z$ & $z$ \\
\hline 0 & 0 & 0 & $C$ & $A$ & $E$ & $v$ & $y$ & $y$ \\
\hline 0 & 0 & 0 & $A$ & $A$ & $A$ & $v$ & $x$ & $x$ \\
\hline 0 & 0 & 0 & $B$ & $A$ & $D$ & $v$ & $w$ & $w$ \\
\hline 0 & 0 & 0 & 0 & 0 & 0 & $A$ & $v$ & $v$ \\
\hline 0 & 0 & 0 & 0 & 0 & 0 & 0 & $\alpha$ & $\alpha$ \\
\hline 0 & 0 & 0 & 0 & 0 & 0 & 0 & 0 & 0 \\
\hline
\end{tabular}

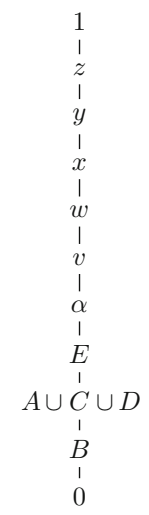

Fig. 4 The tomonoid from Example 4.7

Example 4.7 The next example is based on the work of Kozák [9]. Let $S=$ $\{\dot{0}, v, w, x, y, z, 1\}$ be the seven-element chain, and define a product on $S$ according to the table in Fig. 4(left). We determine the one-element $(1,1)$-coextensions. The $\sim$ classes are indicated in Fig. 4(middle). This time, the equivalence relation $\sim$ does not coincide with $\approx \sim$. Indeed, we have that $(x, w) \preccurlyeq(y, w) \preccurlyeq(y, x) \sim(x, w)$, consequently $(x, w) \sharp_{\sim}(y, w) \sharp_{\sim}(x, w)$, that is, $(x, w) \approx_{\sim}(y, w)$. Similarly, $(x, w) \approx \sim(w, y)$ and we conclude that the union of the $\sim$-classes $A, C$, and $D$, forms a single $\approx \sim$-class. Hence $\approx \sim$ is in this case strictly coarser than $\sim$.

The interval $[0, \alpha]$ consists of five elements only, the Hasse diagram of the poset reduct of $\mathcal{R}_{1,1}(S)$ is as shown in Fig. 4(right). We see that $S$ possesses four one-element $(1,1)$-coextensions.

Example 4.8 Our last example shows the coextensions of a tomonoid such that the chosen border elements are not both 1 . Let $S=\{\dot{0}, v, w, x, y, z, 1\}$ be the seven-element chain, and define a multiplication according to the table in Fig. 5(left). We determine the one-element $(1, z)$-coextensions. Again, the $\sim$-classes are shown, and we have that $\sim$ coincides with $\approx \sim$. The Hasse diagram of the poset reduct of $\mathcal{R}_{1, z}(S)$ is as shown in Fig. 5(right). We observe that there are five one-element $(1, z)$-coextensions.

Let us now turn to the case that coextensions of the desired kind do not exist. Then the free one-element $\left(\varepsilon_{l}, \varepsilon_{r}\right)$-coextension collapses to $S$. 

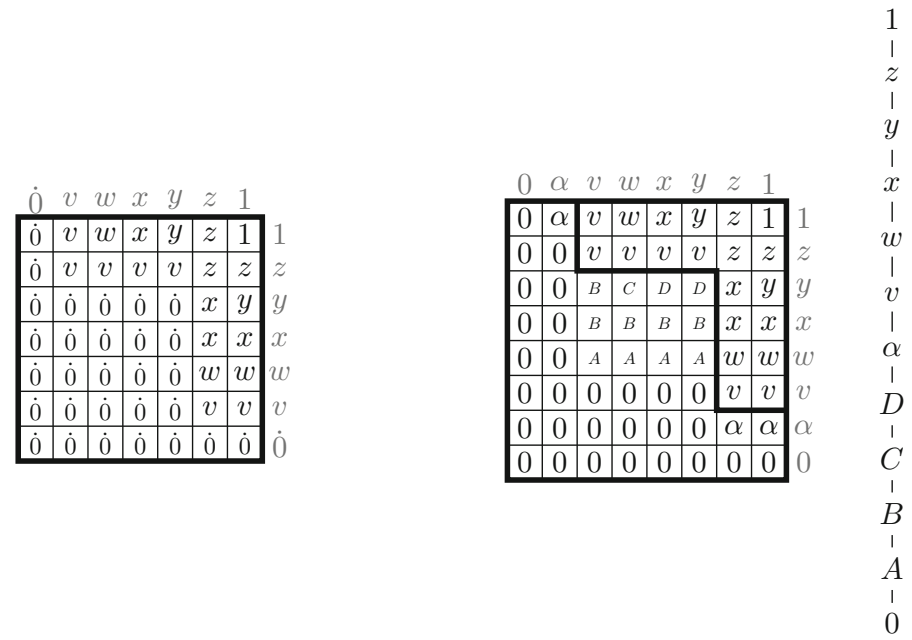

Fig. 5 The tomonoid from Example 4.8

Theorem $4.9 S$ possesses a one-element $\left(\varepsilon_{l}, \varepsilon_{r}\right)$-coextension if and only if $0 \neq \alpha$ in $\mathcal{R}_{\varepsilon_{l}, \varepsilon_{r}}(S)$.

Proof The "only if" is clear from Proposition 4.2.

To see the "if" part, assume that $0 \neq \alpha$. Let $\sim$ be the equivalence relation on $\mathcal{N}$ specified in Theorem 3.16. By Theorem 4.5, we have $(1,0) \not \approx_{\sim}(1, \alpha)$. Then there is a union $\mathcal{Z}$ of $\sim$-classes such that $\mathcal{Z}$ is a downset not containing $(1, \alpha)$. By Theorem 3.16, it follows that there is a one-element $\left(\varepsilon_{l}, \varepsilon_{r}\right)$-coextension of $S$.

If one-element $\left(\varepsilon_{l}, \varepsilon_{r}\right)$-coextensions of $S$ do not exist, then by Theorem 4.9, $\mathcal{R}_{\varepsilon_{l}, \varepsilon_{r}}(S)$ is isomorphic to $S$. If such coextensions do exist, they are easily determined from $\mathcal{R}_{\varepsilon_{l}, \varepsilon_{r}}(S)$. As we will now see, it is indeed enough to choose a downset of $[0, \alpha]$ that contains 0 but not $\alpha$.

Theorem 4.10 Assume that $0 \neq \alpha$ and let $\varnothing \subset Z \subset[0, \alpha]$ be a downset of $\mathcal{R}_{\varepsilon_{l}, \varepsilon_{r}}(S)$. For $a, b \in \mathcal{R}_{\varepsilon_{l}, \varepsilon_{r}}(S)$, let

$$
a \theta_{Z} b \text { if and only if } a=b \text { or } a, b \in Z \text { or } a, b \in[0, \alpha] \backslash Z \text {. }
$$

Then $\theta_{Z}$ is a congruence on $\mathcal{R}_{\varepsilon_{l}, \varepsilon_{r}}(S)$ and $\mathcal{R}_{\varepsilon_{l}, \varepsilon_{r}}(S) / \theta_{Z}$ is a one-element $\left(\varepsilon_{l}, \varepsilon_{r}\right)$ coextension of $S$.

Up to isomorphism, every one-element $\left(\varepsilon_{l}, \varepsilon_{r}\right)$-coextension of $S$ arises in this way from a unique downset $Z$ of $\mathcal{R}_{\varepsilon_{l}, \varepsilon_{r}}(S)$.

Proof The one-element $\left(\varepsilon_{l}, \varepsilon_{r}\right)$-coextensions of $S$ are in one-to-one correspondence with the sets $\mathcal{Z} \subseteq \mathcal{N}$ as specified in Theorem 3.16. Each such subset $\mathcal{Z}$ induces a congruence by requiring $a b=0$ if $(a, b) \in \mathcal{Z}$, and $a b=\alpha$ if $(a, b) \in \mathcal{N} \backslash \mathcal{Z}$.

By Proposition 4.4, $[0, \alpha]=\{a b:(a, b) \in \mathcal{N}\}$. Hence, by Theorem 4.5, the sets $\mathcal{Z}$ are in a one-to-one correspondence with the downsets $Z$ such that $\varnothing \subset Z \subset[0, \alpha]$. 
Accordingly, each such downset $Z$ induces a congruence $\theta$ by requiring $a b=0$ if $a b \in Z$, and $a b=\alpha$ if $a b \in[0, \alpha] \backslash Z$. The assertions follow.

In other words, the one-element $\left(\varepsilon_{l}, \varepsilon_{r}\right)$-coextensions of $S$ are in a one-to-one correspondence with the downsets of the interval $[0, \alpha]$ of $\mathcal{R}_{\varepsilon_{l}, \varepsilon_{r}}(S)$ that contain 0 but not $\alpha$.

We finally turn to the question how we can tell from the structure of $S$ whether or not at least one one-element $\left(\varepsilon_{l}, \varepsilon_{r}\right)$-coextension exists. We know that if one of $\varepsilon_{l}$ or $\varepsilon_{r}$ equals $\alpha$, also the respective other element equals $\alpha$ and the coextension exists. Otherwise, $\varepsilon_{l}$ and $\varepsilon_{r}$ must be idempotent elements of $S^{\star}$. However, not for every such pair a one-element $\left(\varepsilon_{l}, \varepsilon_{r}\right)$-coextension exists; see [10, Fig. 6].

The problem of an exact criterion, which was left unanswered in [10], turns out to be tricky and we conclude the paper by providing one necessary and one sufficient condition. Here, $\backslash$ and / denote the residuals on $S$; we recall that this means

$$
a \cdot b \leqslant c \text { iff } b \leqslant a \backslash c \text { iff } a \leqslant c / b
$$

for $a, b, c \in s$. Moreover, for a non-zero element $a$ of a finite chain, we denote by pred $a$ the element preceding $a$.

Proposition 4.11 Assume that $S$ possesses a one-element $\left(\varepsilon_{l}, \varepsilon_{r}\right)$-coextension. Then the following holds in $S$ :

$$
\begin{aligned}
& \text { pred } \varepsilon_{r} \leqslant\left(\varepsilon_{l} \backslash \dot{0}\right) \backslash \dot{0}, \\
& \text { pred } \varepsilon_{l} \leqslant \dot{0} /\left(\dot{0} / \varepsilon_{r}\right) .
\end{aligned}
$$

Proof We show the first part only; the second part follows by a dual argument.

Assume that $\left(\varepsilon_{l} \backslash \dot{0}\right) \backslash \dot{0}<\operatorname{pred} \varepsilon_{r}$ holds in $S$. This means $\varepsilon_{l} \backslash \dot{0} \cdot$ pred $\varepsilon_{r}>\dot{0}$. It follows that $b=\varepsilon_{l} \backslash \dot{0}$ and pred $\varepsilon_{r}$ are elements of $S^{\star}$ such that, in $\bar{S}$, we have $b \cdot \operatorname{pred} \varepsilon_{r}>\alpha$. Furthermore, $\varepsilon_{l} \cdot b=\varepsilon_{l} \cdot\left(\varepsilon_{l} \backslash \dot{0}\right)=\dot{0}$ in $S$. Also $\varepsilon_{l} \in S^{\star}$, hence we have $\varepsilon_{l} \cdot b \leqslant \alpha$ in $\bar{S}$. But this is a contradiction: in $\bar{S}$, we have $\left(\varepsilon_{l} \cdot b\right) \cdot \operatorname{pred} \varepsilon_{r} \leqslant \alpha \operatorname{pred} \varepsilon_{r}=0$ and $\varepsilon_{l} \cdot\left(b \cdot \operatorname{pred} \varepsilon_{r}\right) \geqslant \varepsilon_{l} \alpha=\alpha$.

Proposition 4.12 Assume that $\varepsilon_{l}, \varepsilon_{r} \in S^{\star}$ and let $\varepsilon_{m}$ be the smallest non-zero idempotent of $S$. Assume that, for $a, b \in \bar{S}$, we have $a b=\dot{0}$ whenever either $a \leqslant \varepsilon_{l}$ and $b<\varepsilon_{m}$ or $a<\varepsilon_{m}$ and $b \leqslant \varepsilon_{r}$. Then $S$ possesses a one-element $\left(\varepsilon_{l}, \varepsilon_{r}\right)$-coextension.

Proof For $a, b \in \bar{S}$, we define

$$
a \cdot b= \begin{cases}a b & \text { if } a, b \in S^{\star} \text { and } a b \text { exists in } S^{\star}, \\ \alpha & \text { if }(a, b) \in \mathcal{N}, a, b \neq 0, \text { and } a \geqslant \varepsilon_{l} \text { or } b \geqslant \varepsilon_{r} \\ 0 & \text { otherwise. }\end{cases}
$$

We claim that this product makes $\bar{S}$ into a tomonoid. The compatibility of the order and the fact that 1 is an identity are readily seen.

It remains to show the associativity. Let $a, b, c \in \bar{S}$; we have to show that $(a \bullet b) \bullet c=$ $a \cdot(b \cdot c)$. We distinguish two cases. 
Case 1. Let $(a \bullet b) \bullet c \in S^{\star}$. Then we have $a \bullet(b \bullet c)=a b c=a \bullet(b \bullet c)$.

Case 2. Let $(a \cdot b) \bullet c \notin S^{\star}$, that is, $(a \bullet b, c) \in \mathcal{N}$. Then also $(a, b \bullet c) \in \mathcal{N}$. We have to show that $(a \cdot b) \bullet c=0$ if and only if $a \bullet(b \bullet c)=0$.

Assume that $(a \bullet b) \bullet c=0$. In case that $a \cdot b=0$ we have $a \bullet(b \bullet c) \leqslant a \cdot b=0$, that is, $a \bullet(b \bullet c)=0$. In case that $c=0$ we have $a \bullet(b \bullet c)=0$ as well. Assume now that $a \bullet b, c \neq 0$. Then $a \bullet b<\varepsilon_{l}$ and $c<\varepsilon_{r}$. We furthermore have that $a<\varepsilon_{m}$ or $b<\varepsilon_{m}$ or $c<\varepsilon_{m}$ because otherwise it would follow that $(a \bullet b) \cdot c \geqslant \varepsilon_{m}$. If $a<\varepsilon_{m}$, we have $a<\varepsilon_{l}$ and $b \bullet c \leqslant c<\varepsilon_{r}$ and hence $a \cdot(b \bullet c)=0$. If $b<\varepsilon_{m}$, then $b<\varepsilon_{l}$ and hence $a \cdot(b \bullet c)=a \bullet 0=0$. Finally, if $c<\varepsilon_{m}$, we conclude from $a \cdot b<\varepsilon_{l}$ that $a<\varepsilon_{l}$ or $b<\varepsilon_{l}$, and we conclude in both cases $a \bullet(b \bullet c)=0$ again. Similarly, we see that $a \cdot(b \cdot c)=0$ implies $(a \bullet b) \bullet c=0$.

We have shown the associativity of the product, thus $\bar{S}$ is indeed a tomonoid. Clearly, $\bar{S} / \alpha$ is isomorphic to $S$.

\section{Conclusion}

We have proposed in this paper a new perspective on the problem of how to determine the one-element Rees coextensions of finite negative tomonoids. Given a finite, negative tomonoid $S$, the pomonoid $\mathcal{R}(S)$ is the free pomonoid generated by the extended chain $\bar{S}$ subject to conditions that hold in any one-element coextension of $S$. We thus get all coextensions of the latter kind as a quotient of $\mathcal{R}(S)$. The determination of the relevant congruence can most easily be done in a two-stage process: for elements $\varepsilon_{l}, \varepsilon_{r}$, which correspond to idempotent elements of $S$, we associate the pomonoid $\mathcal{R}_{\varepsilon_{l}, \varepsilon_{r}}(S)$, which is a quotient of $\mathcal{R}(S)$ and from which to get the actual one-element coextensions is straightforward.

Possibilities to elaborate on our work are numerous. A motivation of the present work has been the creation of a framework in which the generalisation of our method could be facilitated. In particular, we should get along without the condition of negativity. The situation has turned out to be tricky, it is nonetheless worth to be investigated. A possibly less difficult problem might be the generalisation to the case of an arbitrary instead of a total order.

Acknowledgements Open access funding provided by Johannes Kepler University Linz. The authors were supported by the Austrian Science Fund (FWF): Project I 1923-N25 and the Czech Science Foundation (GAČR): Project 15-34697L (New perspectives on residuated posets). In addition, M. P. was supported by the Czech Science Foundation (GAČR): Project GJ15-07724Y.

Open Access This article is distributed under the terms of the Creative Commons Attribution 4.0 International License (http://creativecommons.org/licenses/by/4.0/), which permits unrestricted use, distribution, and reproduction in any medium, provided you give appropriate credit to the original author(s) and the source, provide a link to the Creative Commons license, and indicate if changes were made.

\section{References}

1. Al Subaiei, B.: Unitary posets and amalgamations of pomonoids. Ph.D. thesis, University of Southampton (2014) 
2. Bulman-Fleming, S., Gutermuth, D., Gilmour, A., Kilp, M.: Flatness properties of S-posets. Commun. Algebra 34, 1291-1317 (2006)

3. Evans, K., Konikoff, M., Madden, J.J., Mathis, R., Whipple, G.: Totally ordered commutative monoids. Semigroup Forum 62, 249-278 (2001)

4. Gabovich, E.Y.: Fully ordered semigroups and their applications. Russ. Math. Surv. 31, 147-216 (1976)

5. Galatos, N., Jipsen, P., Kowalski, T., Ono, H.: Residuated Lattices. An Algebraic Glimpse at Substructural Logics. Elsevier, Amsterdam (2007)

6. Hájek, P.: Metamathematics of Fuzzy Logic. Kluwer Academic Publisher, Dordrecht (1998)

7. Howie, J.M.: An Introduction to Semigroup Theory. Academic Press, London (1976)

8. Kehayopulu, N., Tsingelis, M.: Ideal extensions of ordered semigroups. Commun. Algebra 31, 49394969 (2003)

9. Kozák, J.: Algoritmus generující operace ve vícehodnotové logice (Algorithm to generate operations in many-valued logic, in Czech). Bachelor thesis, Czech Technical University, Prague (2017)

10. Petrík, M., Vetterlein, T.: Rees coextensions of finite, negative tomonoids. J. Log. Comput. 27, 337-356 (2017)

11. Poinsot, L., Duchamp, G.H.E., Tollu, C.: Partial monoids: associativity and confluence. J. Pure Appl. Math. Adv. Appl. 3, 265-285 (2010) 